\title{
Joule Heating Effect on MHD Free Convection and Mass Transfer Flow Over a Vertical Plate with Ion-Slip Current in Presence of Rotating System
}

\section{Jamal Hossain ${ }^{1}$ and M. Delowar Hossain ${ }^{2}$}

${ }^{1}$ Department of Mathematics1, Harganga College, Munsigong-1523, Bangladesh

${ }^{2}$ Department of Mathematics, Chittagong University of Engineering \& Technology

Chittagong-4349, Bangladesh

${ }^{2}$ Corresponding author: delowar_cuet@yahoo.com

Received 1 April 2018; accepted 6 June 2018

\begin{abstract}
The effects of Joule heating on MHD free-convection and mass transfer flow over a vertical porous plate with ion-slip currents in a rotating system are investigated. The finite difference method is used as main tool for the numerical approach. The governing equations are derived in the boundary layer and considering Boussinesqs' approximations. The equations with boundary conditions are converted using usual transformations. The effects of magnetic parameter, ion-slip parameter, Eckert number and other involved parameters on the velocity, temperature and concentration field have been studied extensively and represented graphically. It is observed that for different values of the magnetic parameter, the primary velocity distribution decreases while temperature, concentration distribution increases
\end{abstract}

Keywords: MHD, Ion-slip current, Rotation, Joule heating, Permeability, Eckert number.

AMS Mathematics Subject Classification (2010): 76Mxx

\section{Introduction}

The natural convection flow on a vertical surface embedded in porous media occurs in many important engineering and Geophysical applications such as geothermal reservoirs, drying of porous solids, thermal insulation, enhanced oil recovery, packedbed catalytic reactors, cooling of nuclear reactors, and underground energy transport. Hall and ion-slip currents are important and they have a marked effect on the magnitude and direction of the current density and consequently on the magnetic force term. The rotating hydrodynamic flows of incompressible fluids in the presence of porous boundaries occur in MHD power generators, including magnetically such study is also useful in metallurgy. Study of the interaction of Coriolis force with electromagnetic force in porous media is important in some geophysical and astrophysical problems, since many astronomical bodies, posses magnetic field and fluid interiors.

Ferdows et al. [1] investigated the effects of Hall and ion-slip currents on free convective heat transfer flow past a vertical plate considering slip conditions. Steady motions of electrically conducting viscous fluids through a porous medium in presence of 


\section{Jamal Hossain and M. Delowar Hossain}

magnetic field, which are of interest in many scientific and engineering domains, have attracted considerable attention of many investigators. Vidyasagar et al. [2] studied heat and mass transfer effects on MHD boundary layer flow over a moving vertical porous plate. Rajput and Mohammad [3] investigated rotation effect on unsteady MHD flow past an impulsively started vertical plate with variable temperature in porous medium. Das et al. [4] studied Hall effects on unsteady hydromagnetic flow past an accelerated porous plate in a rotating system. Singh and Madhab [5] considered MHD Free Convective Heat and Mass transfer of fluid flow past a moving variable surface in porous media. Quader and Alam [6] investigated the effects of Hall current and viscous dissipation on MHD free convection fluid flow in a rotating system. Raghunath et al.[7] presented Hall Effects on MHD Convective rotating flow of through a porous medium past infinite vertical plate. Nisat Nowroz Anika et al. [8] investigated Hall current effects on magnetohydrodynamics fluid over an infinite rotating vertical porous plate embedded in unsteady laminar flow. Wahiduzzaman et al.[9] studied unsteady MHD-free convection flow past from a rotating vertical plate with the influence of hall and ion-slip current. Very recently, the viscous and Joules dissipation and internal heat generation was taken into account in the energy equation. The effects of viscous dissipation and Joule heating are usually characterized by the Eckert number and the product of the Eckert number and magnetic parameter, respectively and both have a very important part in geophysical flow and in nuclear engineering that was studied by Alim et al [10] . Abdou[11] studied the effects of MHD and Joule heating on free convective boundary layer with a variable plate temperature in a porous medium. Jaber [12] considerd the influence of hall current and viscous dissipation on MHD convective heat and mass transfer in a rotating porous channel with Joule heating. Raju and Srinivasa [13] considered Joule heating effect on MHD free convective heat absorbing/generating viscous dissipative Newtonian fluid with variable temperature. Subbanna et al.[14] discussed MHD free convective flow through porous medium past an infinite vertical plate. Lakshmannal and Venkateswarlu [15] investigated unsteady MHD convective flow of an incompressible viscous fluid through porous medium over a vertical plate.

In this regards our aim is to investigate the Joule heating effect on unsteady MHD free convective heat and mass transfer flow through a vertical porous plate with ion-slip currents in a rotating system. The effects of various emerging parameters on the velocity, temperature and concentration field are discussed graphically in details.

\section{Governing equations}

The two dimensional unsteady flow of an electrically conducting incompressible viscous fluid past semiinfinite vertical porous plate has been considered. The flow is assumed to be in the $x$-axis which is taken along the plate in the upward direction and $y$-axis is normal to it. Initially the fluids as well as the plate are at rest but for time $t>0$ the whole system is allowed to rotate with a constant angular velocity $\Omega$ about the $y$ axis. Both the plate and the fluid are maintained initially at the same temperature. Also it is assumed

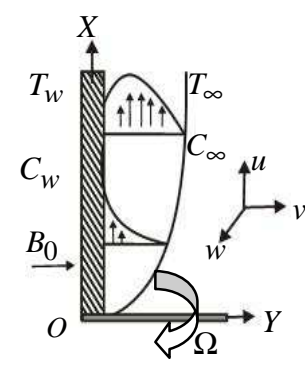

Figure 1: Physical configuration and coordinate system 
Effect of ion-slip current on MHD free convection and mass transfer flow over.

that the temperature of the plate and spices concentration are raised to $T_{w}\left(>T_{\infty}\right)$ and $C_{w}\left(>C_{\infty}\right)$ respectively. The physical configuration of the problem is shown in Fig.1. A strong magnetic field is applied in the $y$-direction. The uniform magnetic field strength $B_{0}$ can be taken as $\mathbf{B}=\left(0, B_{0}, 0\right)$. However, for such a fluid, the ion-slip current will significantly affected the flow in presence of large magnetic fields. The induced magnetic field is neglected, since the magnetic Reynolds number of a partially-ionized fluid is very small. The equation of conservation of electric charge $\bar{\nabla} \cdot \mathbf{J}=0$ gives $j_{y}=$ constant because the direction of propagation is considered only along $y$-axis and $\mathbf{J}$ does not have any variation along the $y$-axis. Since the plate is electrically non-conducting, the constant is zero i.e. $j_{y}=0$ at the plate and everywhere. The equations which govern the flow under the above consideration and Boussinesq's approximation are as follows:

The continuity equation: $\quad \frac{\partial u}{\partial x}+\frac{\partial v}{\partial y}=0$

Momentum equations;

$$
\begin{aligned}
& \frac{\partial u}{\partial t}+u \frac{\partial u}{\partial x}+v \frac{\partial u}{\partial y}=v \frac{\partial^{2} u}{\partial y^{2}}+g \beta_{T}\left(T-T_{\infty}\right)+g \beta_{c}\left(C-C_{\infty}\right)+2 \Omega w-\frac{v}{k} u \\
& -\frac{\sigma_{e} B_{0}^{2}}{\rho\left(\alpha_{e}^{2}+\beta_{e}^{2}\right)}\left(\alpha_{e} u+\beta_{e} w\right) \\
& \frac{\partial w}{\partial t}+u \frac{\partial w}{\partial x}+v \frac{\partial w}{\partial y}=v \frac{\partial^{2} w}{\partial y^{2}}-2 \Omega u-\frac{v}{k} w+\frac{\sigma_{e} B_{0}^{2}}{\rho\left(\alpha_{e}^{2}+\beta_{e}^{2}\right)}\left(\beta_{e} u-\alpha_{e} w\right)
\end{aligned}
$$

Energy equation;

$$
\frac{\partial T}{\partial t}+u \frac{\partial T}{\partial x}+v \frac{\partial T}{\partial y}=\frac{\kappa}{\rho c_{p}} \frac{\partial^{2} T}{\partial y^{2}}+\frac{\sigma_{e} B_{0}^{2}}{\rho c_{p}\left(\alpha_{e}^{2}+\beta_{e}^{2}\right)}\left(u^{2}+w^{2}\right)
$$

Concentration equation;

$$
\frac{\partial C}{\partial t}+u \frac{\partial C}{\partial x}+v \frac{\partial C}{\partial y}=D_{m} \frac{\partial^{2} C}{\partial y^{2}}
$$

The initial and boundary conditions for the problems are;

$$
\begin{aligned}
& t \leq 0, u=0 v=0, w=0, T=T_{\infty}, C=C_{\infty} \text { everywhere } \\
& t>0, u=0 v=0, w=0, T=T_{\infty}, C=C_{\infty} \text { at } \quad x=0 \\
& u=U_{0} v=0, w=0, T=T_{w}, C=C_{w} \quad \text { at } \quad y=0 \\
& u=0, v=0, w=0, T=T_{\infty}, C=C_{\infty} \quad \text { as } \quad y \rightarrow \infty
\end{aligned}
$$

where $\alpha_{e}=1+\beta_{e} \beta_{i}, \beta_{e}$ (Hall parameter), $\beta_{i}$ (ion-slip parameter), $\beta_{T}$ (volumetric coefficient of thermal expansion), $\beta_{c}$ (concentration expansion co-efficient), $\Omega$ (angular velocity), $g$ (acceleration due to gravity), $v$ (Kinematic viscosity), $\rho$ (fluid density), $k$ (permeability of the porous medium), $c_{p}$ (Specific heat at constant pressure), $\kappa$ (Thermal 


\section{Jamal Hossain and M. Delowar Hossain}

conductivity), $D_{m}$ (Co-efficient of mass diffusivity), $B_{0}$ (uniform magnetic field), $\sigma_{e}$ (Electrical conductivity), $T$ (temperature in the boundary layer), $T_{\infty}$ (temperature outside the boundary layer), $C$ (concentration in the boundary layer), $C_{\infty}$ (concentration outside the boundary layer), $t$ (dimensional time), $u, v, w$ are velocities in coordinates $x, y, z$.

\section{Mathematical formulation}

The problem is simplified by writing the equations in the non-dimensional form. Now introduce the following non-dimensional quantities

$$
X=\frac{x U_{0}}{v}, Y=\frac{y U_{0}}{v}, U=\frac{u}{U_{0}}, V=\frac{v}{U_{0}}, \tau=\frac{t U_{0}^{2}}{v}, \bar{T}=\frac{T-T_{\infty}}{T_{w}-T_{\infty}}, \bar{C}=\frac{C-C_{\infty}}{C_{w}-C_{\infty}}
$$

Then introducing the dimensionless quantities (8) in equations (1)-(5) respectively, the following dimensionless equations are as follows;

$$
\begin{aligned}
& \frac{\partial U}{\partial X}+\frac{\partial V}{\partial Y}=0 \\
& \frac{\partial U}{\partial \tau}+U \frac{\partial U}{\partial X}+V \frac{\partial U}{\partial Y}=\frac{\partial^{2} U}{\partial Y^{2}}+G_{r} \bar{T}+G_{m} \bar{C}+2 R W-\gamma-\frac{M\left(\alpha_{e} U+\beta_{e} W\right)}{\alpha_{e}^{2}+\beta_{e}^{2}} \\
& \frac{\partial W}{\partial \tau}+U \frac{\partial W}{\partial X}+V \frac{\partial W}{\partial Y}=\frac{\partial^{2} W}{\partial Y^{2}}-2 R U-\gamma W+\frac{M\left(\beta_{e} U-\alpha_{e} W\right)}{\alpha_{e}^{2}+\beta_{e}^{2}} \\
& \frac{\partial \bar{T}}{\partial \tau}+U \frac{\partial \bar{T}}{\partial X}+V \frac{\partial \bar{T}}{\partial Y}=\frac{1}{P_{r}} \frac{\partial^{2} \bar{T}}{\partial Y^{2}}+\frac{M\left(U^{2}+W^{2}\right)}{\alpha_{e}^{2}+\beta_{e}^{2}} \\
& \frac{\partial \bar{C}}{\partial \tau}+U \frac{\partial \bar{C}}{\partial X}+V \frac{\partial \bar{C}}{\partial Y}=\frac{1}{S_{c}} \frac{\partial^{2} \bar{C}}{\partial Y^{2}}
\end{aligned}
$$

The corresponding boundary conditions are as follows;

$t \leq 0, U=0, V=0, W=0, \bar{T}=0, \bar{C}=0 \quad$ everywhere

$t>0, U=0, V=0, W=0, \bar{T}=0, \bar{C}=0 \quad$ at $X=0$

$U=1, V=0, W=0, \bar{T}=1, \bar{C}=1 \quad$ at $Y=0$

$U=0, W=0, \bar{T}=0, \bar{C}=0 \quad$ as $Y \rightarrow \infty$

where $G_{r}=\frac{g \beta_{T}\left(T_{w}-T_{\infty}\right) v}{U_{0}^{3}}$ (Grashof number), $G_{m}=\frac{g \beta_{c}\left(C_{w}-C_{\infty}\right) v}{U_{0}^{3}}$ (modified Grashof

number), $\quad M=\frac{\sigma_{e} B_{0}^{2} v}{\rho U_{0}^{2}}$ (magnetic parameter), $P_{r}=\frac{\rho v c_{p}}{\kappa}$ (Prandtl number), $S_{c}=\frac{v}{D_{m}}$

(Schmidt number), $R=\frac{\Omega v}{U_{0}^{2}}$ (rotation parameter), $\quad \gamma=\frac{v^{2}}{k U_{0}^{2}}$ (permeability parameter), $E_{c}=\frac{U_{0}^{2}}{c_{p}\left(T_{w}-T_{\infty}\right)}($ Eckert number $)$ 
Effect of ion-slip current on MHD free convection and mass transfer flow over.

\section{Solution technique}

The governing second order non-linear coupled dimensionless partial differential equations have been solved numerically with the associated boundary conditions. The explicit finite difference method has been used to solve the coupled equations (9)-(13) with boundary conditions (14)-(15).To obtain the difference equations the region of the flow is divided into a grid or mesh of lines parallel to $X$ and $Y$ axes, where $X$-axis is taken along the plate and $Y$-axis is taken normal to the plate. Here the plate height $X_{\max }(95.0)$ is considered i.e. $X$ varies from

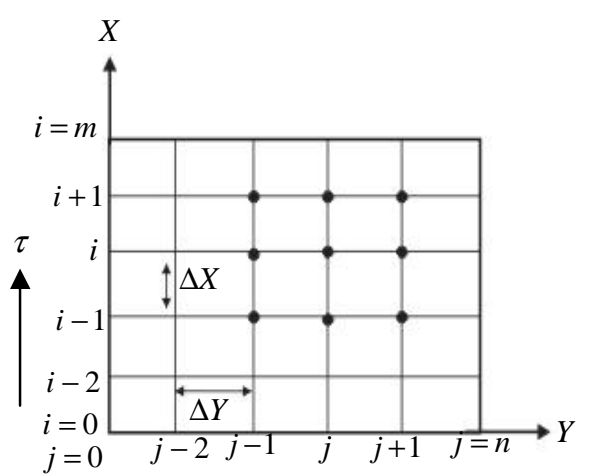

Figure 2: Finite difference grid

0.0 to 95.0 and assumed $Y_{\max }(65.0)$ as corresponding $Y \rightarrow \infty$ i.e. $Y$ varies from 0.0 to 65.0. There are $m=250.0$ and $n=250.0$ grid spacing in the $X$ and $Y$ directions respectively and taken as follows $\Delta X=0.38(0 \leq X \leq 95)$ and $\Delta Y=0.26(0 \leq Y \leq 65)$ with the smaller time step $\Delta \tau=0.005$.

\section{Results and discussion}

The physical situation of the above model, the velocity profiles in $x$ and $z$ components are commonly known as the primary and secondary velocities. The numerical results has been carried out for dimensionless primary velocity $(U)$, secondary velocity $(W)$, temperature $(\bar{T})$, species concentration $(\bar{C})$, local shear stresses in $x$-axis $\left(\tau_{L U}\right)$, local shear stresses in $z$-axis $\left(\tau_{L W}\right)$, local Nusselt numbers $\left(N_{u L}\right)$ for various values of the material parameters such as ion-slip parameter $\left(\beta_{i}\right)$, magnetic parameter $(M)$, rotation parameter $(R)$, Prandtl number $\left(P_{r}\right)$, Schmidt number $\left(S_{c}\right)$, permeability parameter $(\gamma)$, Eckert number $\left(E_{c}\right)$. The values for the parameters are chosen arbitrarily in most cases. Some standard values for of the Prandtl number $\left(P_{r}\right)$ is considered because of the physical importance. These are $P_{r}=0.71$ corresponds to air, $P_{r}=1.0$ corresponds to electrolyte solution such as salt water and $P_{r}=1.63$ corresponds to glycerin at $50^{\circ} \mathrm{C}$ and the value of Schmidt number is considered $S_{c}=0.60$ which represent specific condition of flow ( 0.60 corresponds to water vapor). The importance of cooling problem in nuclear engineering in connection with the cooling of reactors, the values of $G_{r}$ and $G_{m}$ are taken positive. Throughout the calculations the values of $G_{r}$ and $G_{m}$ are taken very large ( $G_{r}=5.0$ and $G_{m}=2.0$ ). Form Figures 3-4, it is seen that the primary velocity ( $U$ ), local shear stresses in $x$-axis $\left(\tau_{L U}\right)$ increase with an increase of ion-slip parameter $\left(\beta_{i}\right)$. This is due to fact that the effective conductivity decreases, which reduces the magnetic resistive force affecting on the primary flow. The secondary velocity $(W)$ ) has decreasing effect with the increase of $\beta_{i}$ which are found in Figures 5. From this figure it is clear that ion-slip parameter $\beta_{i}$ retards the flow which leads to reduction in boundary layer 


\section{Jamal Hossain and M. Delowar Hossain}

thickness. Analyzing the Figure 6, it is clearly seen that the primary velocity $(U)$ profiles increases with the increase $E_{c}$. This is due to the heat energy stored in the liquid because of the frictional heating. The effect of Eckert number on the temperature is shown in Figure 7. The effect of viscous dissipation on flow field is to increase the energy, yielding a greater fluid temperature and as a consequence greater buoyancy force. The increase in the buoyancy force due to an increase in the dissipation parameter enhances the temperature. Local Nusselt numbers $\left(N_{u L}\right)$ has reverse effect which is shown in Figure 8. From Figures 9, it has been seen that the primary velocity $(U)$ decreases whereas the secondary velocity $(W)$ increases with an increase of magnetic parameter in Figures 10. An increase in the value of the magnetic parameter leads to increase in the magnitude of the Lorentz force which serves to retard the primary velocity. The result of Figures 10 indicates that the resulting Lorentzian body force will not act as a drag force, but as an aiding body force. This will serve to accelerate the secondary fluid velocity. From Figures $11-12$, it is seen that the primary velocity $(U)$, local shear stresses in $x$-axis $\left(\tau_{L U}\right)$ decrease with an increases of Prandtl number $\left(P_{r}\right)$. This is because in the free convection the plate velocity is higher than the adjacent fluid velocity and the momentum boundary layer thickness decreases. From Figure 13, it is observed that the temperature distributions decrease with increasing values of Prandtl number. This is consistent with the well-known fact that the thermal boundary layer thickness decreases with increasing values of Prandtl number.

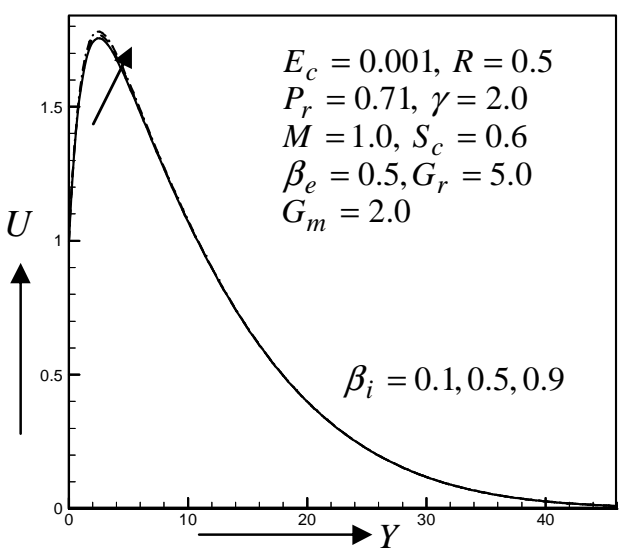

Figure 3: Primary velocity profiles for different values of $\beta_{i}$

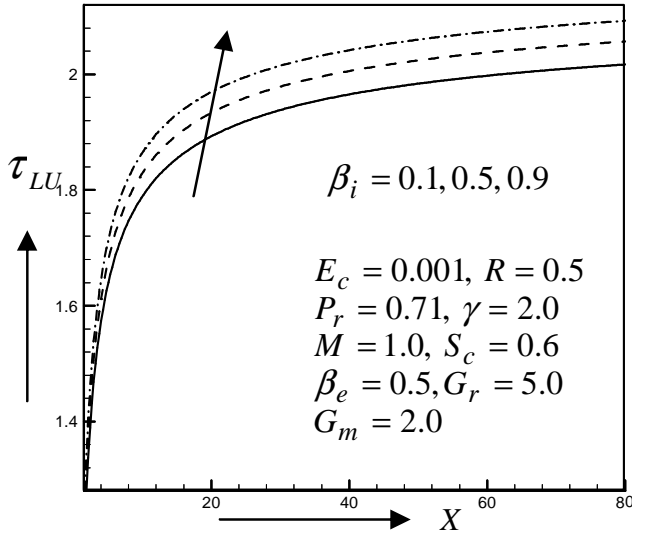

Figure 4: Local Shear stress in $x$-axis for different values of $\beta_{i}$ 
Effect of ion-slip current on MHD free convection and mass transfer flow over.

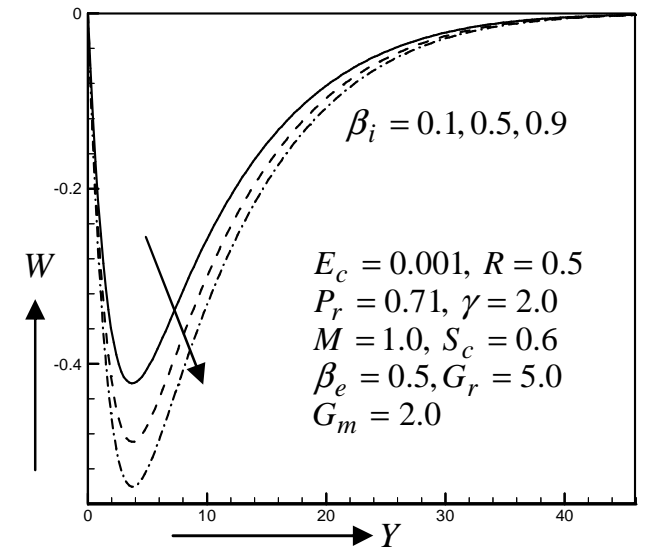

Figure 5: Secondary velocity profiles for different values of $\beta_{i}$

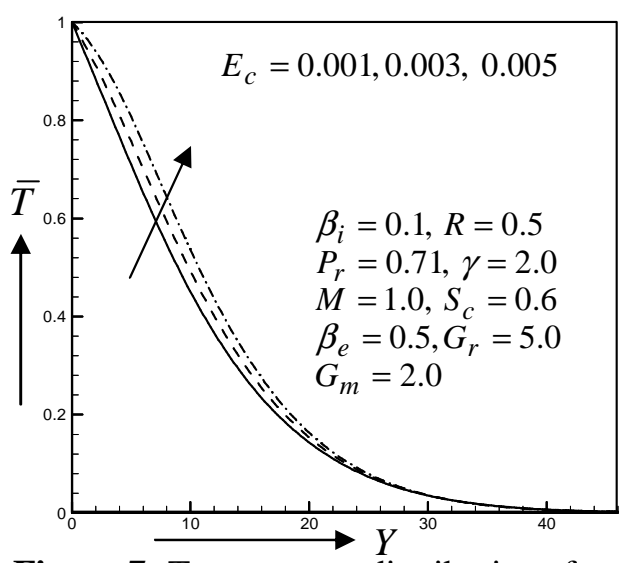

Figure 7: Temperature distributions for different values of $E_{c}$

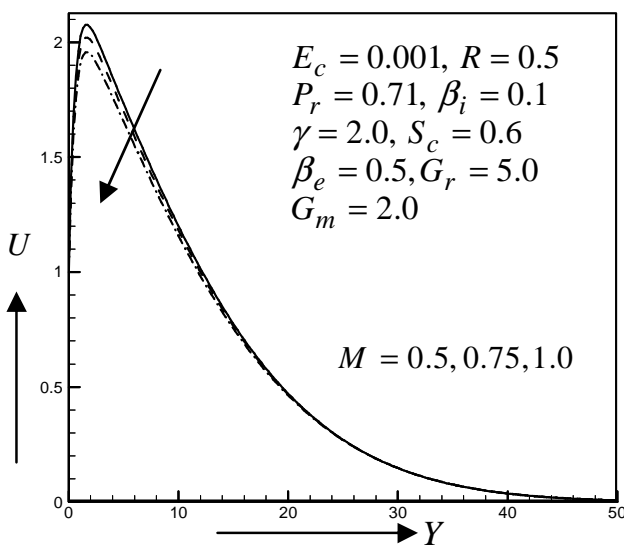

Figure 9: Primary velocity profiles for different values of $M$

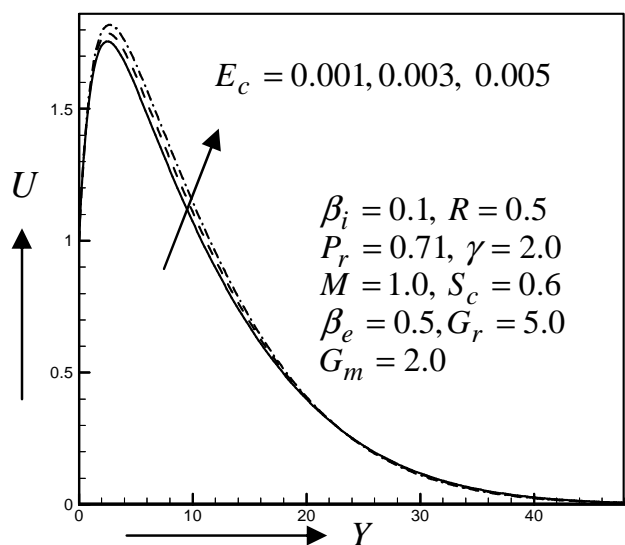

Figure 6: Primary velocity profiles for different values of $E_{c}$

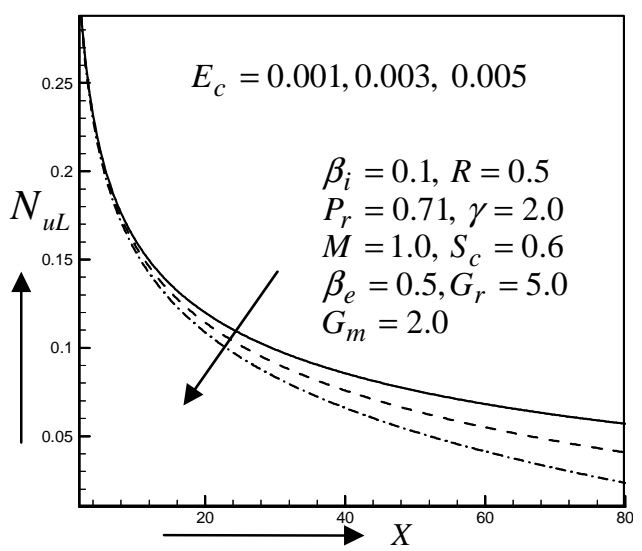

Figure 8: Local Nusselt number for different values of $E_{c}$

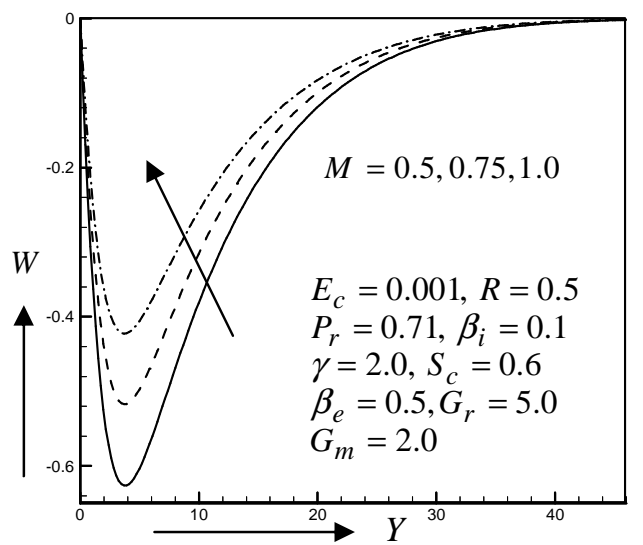

Figure 10: Secondary velocity profiles for different values of $M$ 
M. Jamal Hossain and M. Delowar Hossain

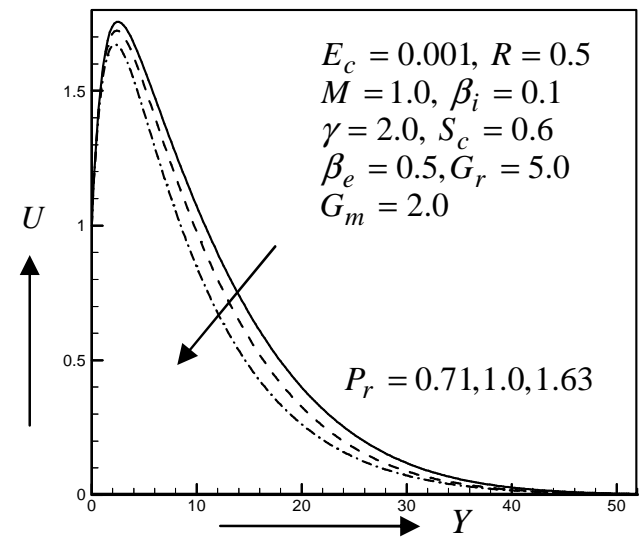

Figure 11: Primary velocity profiles for different values of $P_{r}$

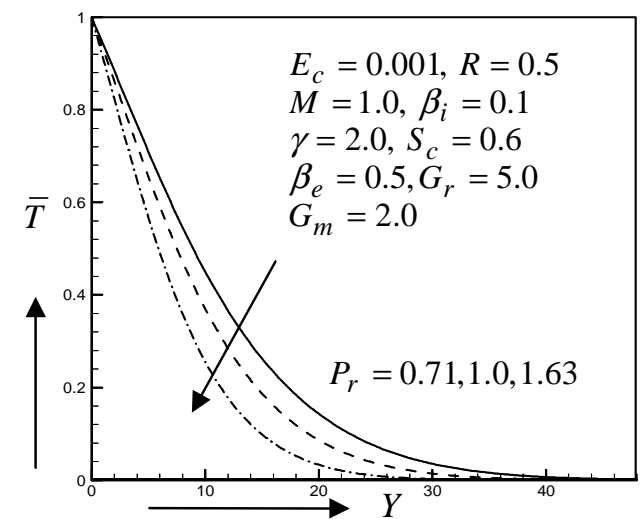

Figure 13: Temperature distributions for different values of $P_{r}$

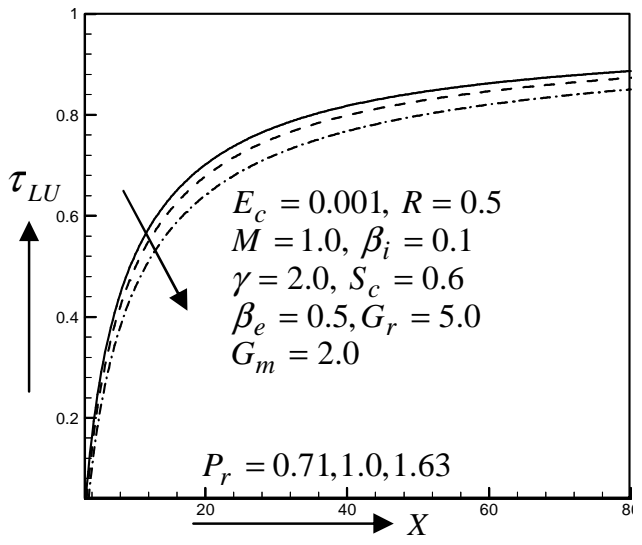

Figure 12: Local Shear stress in $x$-axis for different values of $P_{r}$

\section{Conclusion}

The Effect of ion-slip current on MHD free convection and mass transfer flow over a vertical porous plate in presence of rotating system have been studied numerically. The effects of governing physical parameters on the primary velocity, secondary velocity, temperature as well as local shear stress in $\mathrm{x}$-and $\mathrm{z}$ - axes, local Nusselt number are presented in graphically.

- The primary velocity, local shear stress in $\mathrm{x}$-axis is increased while the secondary velocity is decreased with increasing values of ion-slip current parameter.

- The primary velocity, local shear stress in x-axis, temperature distributions are increased where as local Nusselt number is decreased with increasing values of Eckert number.

- The primary velocity is decreased but the secondary velocity is increased with increasing values of magnetic parameter.

- The primary velocity, local shear stress in $\mathrm{x}$-axis, temperature distributions are 
Effect of ion-slip current on MHD free convection and mass transfer flow over.

decreased whereas local Nusselt number is increased with the increasing values of Prandtl number.

Acknowledgement. The authors wish to convey their thanks to the reviewers for very useful comments and suggestions in improving the paper.

\section{REFERENCES}

1. M.Ferdows, Esrat Jahan, M.M.Hamad and Masahiro OTA, Effects of Hall and ionslip currents on free convective heat transfer flow past a vertical plate considering slip conditions, Canadian Journal on Science and Engineering Mathematics, 2(2) (2011) 70-76.

2. G.Vidyasagar, B.Ramana, P.Bala and Anki Reddy, Heat and mass transfer effects on MHD boundary layer flow over a moving vertical porous plate, Journal of Global Research in Mathematical Archives, 1(2) (2013) 49-62.

3. U.S.Rajput and Mohammad Shareef, Rotation effect on unsteady MHD flow past an impulsively started vertical plate with variable temperature in porous medium, International Journal of Mathematical Archive, 7(2) (2016) 148-153.

4. S.Das, S.K.Guchhait and R.N.Jana, Hall effects on unsteady hydromagnetic flow past an accelerated porous plate in a rotating system, Journal of Applied Fluid Mechanics, 8(3) (2015) 409- 417.

5. Ashok Kumar Singh and Benu Madhab, MHD free convective heat and mass transfer of fluid flow past a moving variable surface in porous media, International Journal of Engineering Trends and Technology, 4(4) (2013) 1151-1157.

6. Abdul Quader and Md. Mahmud Alam, Effects of Hall current and viscous dissipation on MHD free convection fluid flow in a rotating system, AMSE JournalsSeries: Modelling B, 83 (2014) 110-128.

7. K.Raghunath, R.Siva Prasad and G.S.S.Raju, Hall effects on MHD convective otating flow of through a porous mediumpast infinite vertical plate, Annals of Pure and pplied Mathematics, 16(2)(2018) 353-263.

8. Nisat Nowroz Anika, Md. Mainul Hoque and Nazmul Islam, Hall current effects on magnetohydrodynamics fluid over an infinite rotating vertical porous plate embedded in unsteady laminar flow, Annals of Pure and Applied Mathematics, 3(2) (2013) 189200.

9. M. Wahiduzzaman, Gopal Chandra Mazumder, Md. Tajul Islam, A. Sarker and M.S. Uddin, Unsteady MHD-free convection flow past from a rotating vertical plate with the influence of hall and ion-slip current, Annals of Pure and Applied Mathematics, 9(1) (2015) 91-106.

10. M.A.Alim, Md. Mahmud Alam Abdullah Al-Mamun, Joule heating effect on the coupling of conduction with magnetohydrodynamic free convection flow from a vertical plate, Nonlinear Analysis Modelling and Control, 3(3) (2007) 307-316.

11. Mohammed Modather Mohammed Abdou, Effects of MHD and Joule heating on free convective boundary layer with a variable plate temperature in a porous medium, Applied Mathematical Sciences, 11(36) (2017) 1765-1777.

12. Khaled K. Jaber, Influence of hall current and viscous dissipation on MHD convective heat and mass transfer in a rotating porous channel with Joule heating, American Journal of Mathematics and Statistics, 5(5) (2015) 272-284. 
M. Jamal Hossain and M. Delowar Hossain

13. A B Madhu Mohana Raju and Dr G.Srinivasa, Joule heating effect on MHD free convective heat absorbing/generating viscous dissipative Newtonian fluid with variable temperature, International Journal of Research-Granthaalayah, 5(4) (2017) 22-32.

14. K.Subbanna, S. Gouse Mohiddin and R.Bhuvana Vijaya, MHD Free Convective Flow Through Porous Medium Past an Infinite Vertical Plate, Annals of Pure and Applied Mathematics, 16(2) (2018) 419-428.

15. B.Lakshmannal and S.Venkateswarlu, Unsteady MHD convective flow of an incompressible viscous fluid through porous medium over a vertical plate, Annals of Pure and Applied Mathematics, 16(2) (2018) 451-460. 